\title{
FUNGAL NEUROLOGICAL SEQUELAE IN A HYPER IMMUNOGLOBULIN E SYNDROME FROM PESHAWAR, PAKISTAN: AN UNUSUAL PRESENTATION
}

\author{
Nadeem ljaz', iDHuma Hanif', (DImtiaz Ur Rehman', (DSohail Daud Khan², iD Osama Sherjeel Khan ${ }^{3}$ \\ ${ }^{1}$ Surgical ICU \& ${ }^{2}$ Department of Neurosurgery, Northwest General Hospital \& Research Centre, ${ }^{3}$ Department of \\ Surgery, Khyber Teaching Hospital, Peshawar, Pakistan
}

\begin{abstract}
Hyper IGE syndrome is one of the primary immunodeficiency syndromes characterized by the presence of abnormally raised serum IGE levels (>2000 IU/ml) with defective humoral and cell mediated immunity presenting in infancy or early childhood. It is a rare disease with only about 200 cases reported in literature. Usually, it is diagnosed clinically and by raised serum IGE levels. Mostly, it presents with recurrent staphylococcal skin abscesses, sinopulmonary infections, and opportunistic mycotic infections due to the immunodeficient state. Rarely, the central nervous system is involved by this condition.

This case study deals with an 11-year-old girl who was diagnosed as a case of hyper IGE syndrome. She presented with low GCS and focal neurological deficits which were attributed to aspergillus fumigatus infection spreading to the brain resulting in multiple abscesses and eventually to death of the patient despite treatment interventions. This case emphasizes the likelihood of dissemination of fungal infections to multiple organs including the brain and the potential role of early neuroimaging to detect such lesions. Moreover, it shows significance of serial imaging in admitted patients particularly with the onset of new and unusual neurological symptoms. With more extensive studies, we may be able to diagnose and treat such infections and their complications on time with better outcomes.

KEY WORDS: Hyper Immunoglobulin E Syndrome; Neuroaspergillosis; Voriconazole; Brain Abscess; Pneumonia. Cite as: Ijaz N, Hanif H, Rehman IU, Khan SD, Khan OS. Fungal neurological sequelae in a hyper immunoglobulin E syndrome from Peshawar, Pakistan: an unusual presentation [case report]. Gomal J Med Sci 2021 Apr-Jun; 19(2):78-82. https://doi.org/10.46903/gjms/19.02.993
\end{abstract}

\section{INTRODUCTION}

Hyper IgE Syndrome (HIES), also known as Job's syndrome, is a primary immunodeficiency and very rare in prevalence worldwide. It is characterized primarily by symptoms of recurrent sinopulmonary infections, skins abscesses particularly staphylococcal, and atopy such as eczema. ${ }^{1}$ Most of these cases are sporadic that occur during infancy or during first few years of life, while some familial cases have also been reported. Laboratory findings include eosinophilia with increased levels of serum $\mathrm{IgE}$ and normal levels of the rest of the immuno-

\section{Corresponding Author:}

Dr. Nadeem ljaz

Medical Officer, Surgical ICU

Northwest General Hospital \& Research Centre

Peshawar, Pakistan

E-mail: nadeemijaz40@gmail.com

Date Submitted: 09-01-2021

Date Revised: $\quad$ 14-03-2021

Date Accepted: 29-03-2021 globulins (IgM, IgG, IgA). Currently, treatment of Hyper IgE syndrome is symptomatic and relies on prophylactic antimicrobials along with great care of skin and connective tissue. ${ }^{2}$ The accurate incidence of disease is not fully known but a rough estimate is between 1 patient in every 0.5 to 1 million individuals. Gender wise distribution is equal. Ethnic diversity is also reported as HIES can be found in Asian, Africans, as well as Caucasians. ${ }^{3}$ Neutrophil counts in patients with HIES are normal in terms of quantity and the neutrophils can engulf and kill microbes normally. However, there is a defect in chemotaxis in neutrophils of these patients that makes them susceptible to recurrent infections. ${ }^{4}$ Moreover, these patients also have an impaired production of cytokine along with minimal inflammation. This causes them to have non-inflamed or cold abscesses. ${ }^{5}$ Cold abscesses are one of the most common and initial presentations in patients with HIES that should increase a clinician's suspicion for further workup. They can be formed in any part of the human body but in patients with 
HIES, tendency for abscess formation is mainly in skin, lungs, brain, and liver. Skin infections start in early life, and apart from abscesses, they include furuncles, lymphadenitis, and cellulitis. The most common species in abscess formation in HIES patient is staphylococcus species which has tendency around neck, face, and the scalp region. Candida albicans is also notorious for abscess formation in these patients skin. ${ }^{6}$ Sometimes, patients with HIES might be suffering from an underlying infection while still report feeling normal and afebrile. ${ }^{7}$ Lung abscesses are the second most common manifestation of patients presenting with HIES. In one of the studies, the mean age found in a European national survey for presentation with first infection was 10.5 months. ${ }^{8}$ Neurological problems presenting in these patients are rare and when they occur, patients present with meningitis, stroke, and aneurysm. Some case reports have also reported brain malignancies occurring in these patients. ${ }^{9}$ Brain abscess in HIES is very rare and usually fatal. They usually present with symptoms consistent with a space occupying lesion where these patients experience nausea, lethargy, vomiting, headache, decreased level of consciousness, and epilepsy..$^{10,11}$ In one study, a patient with hyper IgE syndrome developed brain abscess after hematopoietic cell transplant. ${ }^{12}$ Another reported tuberculous brain abscess in a similar patient. ${ }^{13}$ These rare cases have reported bacterial etiology as the cause of brain abscess. Our case describes a patient with hyper IgE syndrome that developed fungal brain abscess. No such case has been reported in literature before.

\section{CASE PRESENTATION}

This 11 years old girl presented to us in August 2020, who was diagnosed as Hyper IgE Syndrome, also known as Job's syndrome. She presented with vomiting, headache, slurred speech, and had developed sudden onset right sided face, upper limb and lower limb weakness.

The past history is of prime importance to understand this case. This is because it is pertinent to be clear on how the events led to a disseminated fungal infection, ending up in a neurosurgical emergency. The patient had a history of recurrent sore throats since 1.5 years of age, being treated with multiple antibiotics. At the age of three, she developed cervical lymphadenopathy with on off febrile episodes. At that time, an FNAC of her cervical lymph node was done and was reported as chronic granulomatous inflammation. The size of cervical lymph nodes increased and she was empirically started on anti-tuberculous therapy but showed no response and it was discontinued and treated empirically with antibiotics. Her condition improved.

At the age of eight, she had abdominal pain, fever, and weight loss for which a CT abdomen and pel- vis was done. It reported hepatomegaly, hepatic, renal and splenic infiltrates and had abdominal and mediastinal lymphadenopathy. A CT guided biopsy of para-aortic lymph nodes was done which revealed extensive necrotizing lymphoid tissue, vague granulomas, and numerous septate fungal hyphae. The CT brain at that time was normal. The treatment regimen was comprised of IV amphotericin B and oral fluconazole. The fever settled and the abdominal nodes regressed in size in the follow-up scans. A month later, she presented with three weeks history of productive cough. She was started on oral Voriconazole for a period of six months to treat her as a case of disseminated fungal infection. She had follow-up visits in which CT scans were done to monitor disease progression which reported persistent lymphadenopathy. However, clinically she remained well. After course completion, Voriconazole was discontinued. A follow-up CT scan was done after nine months and it reported hepatosplenomegaly, nodular densities in gastro-hepatic ligament, and regressed para-aortic lymph nodes. However, two peripheral lesions were noted in right lower lung lobe. She was recommenced on Voriconazole for another six months and prednisolone was started as 10 $\mathrm{mg}$ twice daily which was tapered down to $5 \mathrm{mg}$ on alternate days over ten months. On her scheduled follow-up, the CT scan showed remission of hepatic nodules and decreased hepatosplenomegaly, thus the treatment was stopped. A repeat CT scan was done after four months which revealed resolution of para-aortic nodes with no pulmonary nodules. At that point, her IgE levels were concerning, therefore, prednisolone was started again with tapering over six months duration.

She presented again after three months with history of productive cough, fever, night sweats, and left sided chest pain. Her $x$-ray was suggestive of parapneumonic effusion. She was given intravenous antibiotics but showed no response. This time again a fungal infection was suspected and intravenous amphotericin B was added to her treatment regimen. Three days later, she presented with vomiting, headache, slurred speech, and had developed sudden onset right sided face, upper limb and lower limb weakness. Her GCS was 13/15. An emergency CT brain without contrast was done. It reported an ill-defined isodense lesion in the left frontoparietal region, midline shift to the right, and dilation of left temporal horn lateral ventricle. (Figure 1-A \& 1-B)

She was admitted to the hospital and was started on intravenous antibiotics, intravenous amphotericin B and supportive treatment. Her right sided plantar was upgoing. She had power of $1 / 5$ in her right upper limb and 2/5 in her right lower limb. C-Reactive Protein was reported as $160 \mathrm{mg} / \mathrm{L}$ and an Erythrocyte sedimentation rate of $95 \mathrm{~mm} / 1^{\text {st }}$ 
hour. To know the extent of disease, MR Brain with gadolinium contrast was done the following day. It reported lesions in the right and left frontal lobes and left basal ganglia suggesting multifocal cerebral abscesses with cerebritis. It also revealed right sided midline shift as well as dilation of atrium and temporal horn of the left lateral ventricle along with abnormal signals and enhancement suggestive of ventriculitis. (Figure 1-C, 1-D)

The patient improved with the given treatment and neurosurgery was consulted. After optimization and discussion with neurosurgeon; left fronto-temporo-parietal decompressive craniectomy with extra-ventricular drain placement was planned and was done on the fifth day of her admission to the hospital. The patient was shifted to the intensive care unit and was kept sedated and relaxed for 24 hours on ventilator. She was weaned off from ventilator and her treatment regimen was continued. In her management plan, galactomannan and alpha-D-glucan level in the cerebrospinal fluid were considered and sent for reporting the same day. Amphotericin B was stopped as the patient developed severe hypokalemia (potassium $=2.4 \mathrm{mmol} / \mathrm{l}$ ) and bradycardia (heart rate $51 / \mathrm{min}$ ). (Figure 2)

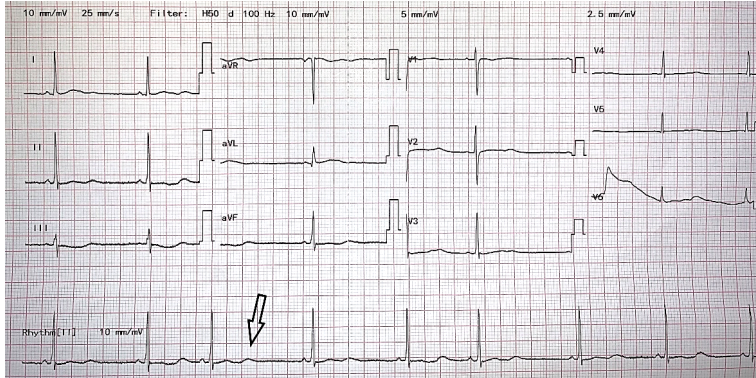

Figure 2: A 12-lead ECG showing bradycardia and delta waves due to hypokalemia in 11-yearold girl with Hyper IGE syndrome from Peshawar, Pakistan

On her $7^{\text {th }}$ post operation day, the patient dropped GCS to 4/15 for which she was intubated. An emergency CT scan was done which reported herniation of brain tissues through cranial vault defect. (Figure $1-E)$

Her intracranial pressure was raised; hence, a second extra-ventricular drain was placed due to blockage of the first one. The left pupil was also dilated and fixed with no response to light. The galactomannan and alpha-D-glucan levels were
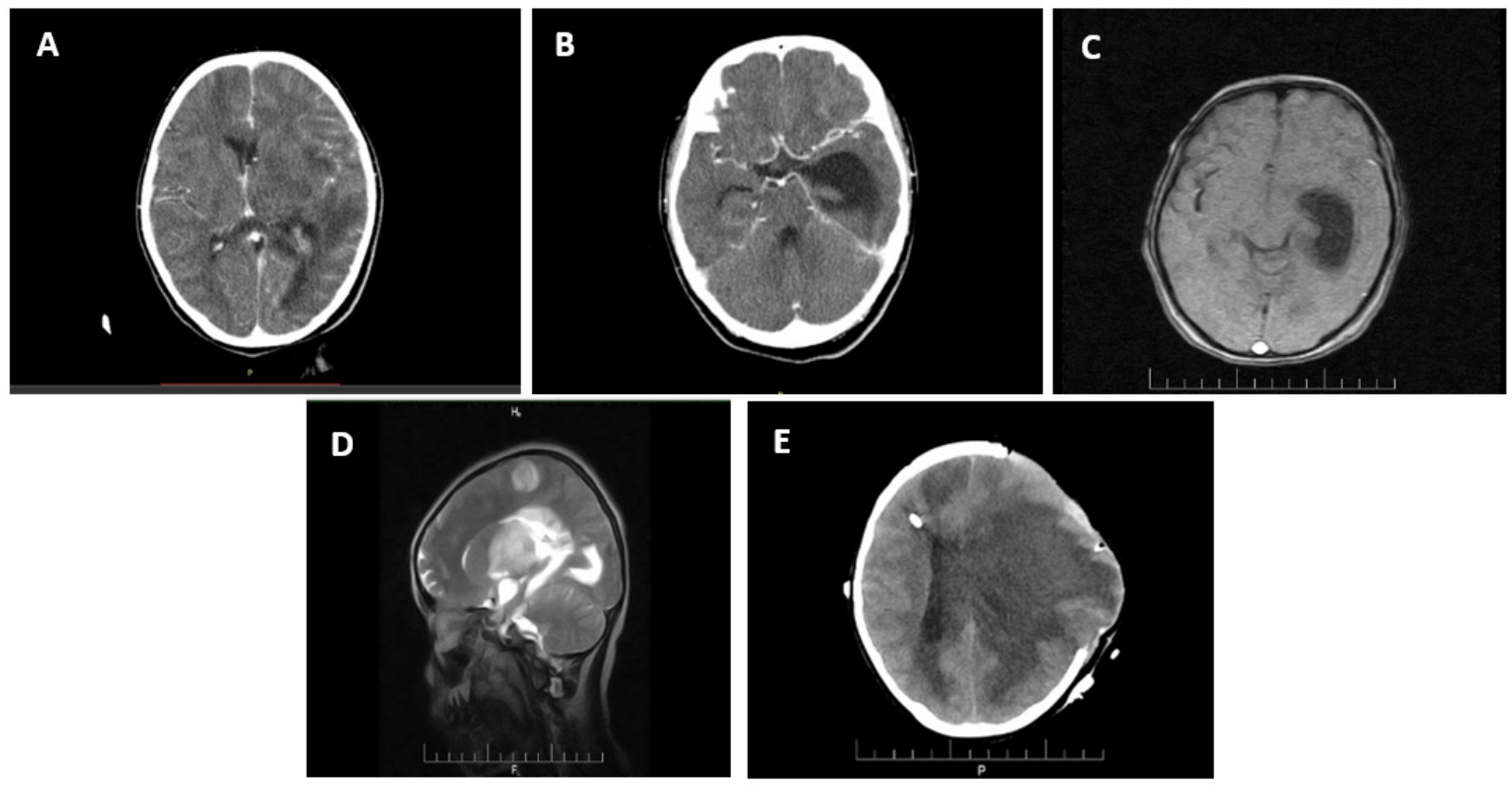

Figure 1: CT brain with contrast axial section showing vasogenic edema in left temporo-parietal region with mass effect in the form of effaced sulci and ipsilateral lateral ventricle with midline shift and slight meningeal enhancement (A). CT brain with contrast axial section showing dilated temporal horn of left lateral ventricle with transependymal seepage and slight midline shift (B). FLAIR MR brain axial section showing dilated temporal horn of left lateral ventricle (C). MR brain T2 weighted sagittal section showing hyper intense lesion in left frontal lobe posterior-superiorly and splenium of corpus collosum (D). CT brain without contrast axial section at the level of body of lateral ventricle showing defect in frontotemporal bone with brain herniation and vasogenic edema with mass effect on ipsilateral lateral ventricle and slight midline shift (E). 
reported positive suggesting a possible aspergillus infection. Voriconazole was added to her treatment regimen this time instead of amphotericin $B$ to avoid hypokalemia. On her ninth day post operation, her both pupils were fixed and dilated with no response to light and had no spontaneous breathing efforts. Brain death tests were performed and unfortunately, she was declared as brain dead.

\section{DISCUSSION}

Job syndrome was first defined by Davis et al. in 1966 as eczema, recurrent skin and pulmonary infections, and eosinophilia. This definition was later on modified in 1972 by Buckley et al. who added elevated lgE levels, hence, the name HIES or Buckley syndrome. Around 200 cases of this syndrome have been described so far in literature. ${ }^{2} \cdot 5$ This rare primary immunodeficiency is classically characterized by an extremely elevated serum IgE levels, eczema, and recurrent skin and pulmonary infections. It is also associated with connective tissue and skeletal abnormalities. The affected individuals have a typical facial appearance and delayed or failure to shed primary teeth. Skeletal abnormalities include joint hyperextensibility, scoliosis, craniosynostosis, and osteopenia with pathologic fractures. The humoral and cellular components of the immune system are usually defective. Staphylococcus aureus is usually responsible for recurrent skin abscesses and pneumonias. Acute pneumonias can give rise to pneumatoceles which may later become superinfected with gram-negative bacteria and fungal opportunistic infections. ${ }^{1,14}$ There are two variants of hyper IgE syndrome. An autosomal dominant which usually involves mutation in STAT 3 gene and cause HIES in approximately $60 \%$ of the patients. ${ }^{2}$ The autosomal recessive is due to mutations in Tyrosine kinase 2 gene and is uncommon. Both forms involve defective immune system and elevated levels of immunoglobulin E in the blood. However, other immunoglobulins levels are usually normal but can present with one or more of these deficiencies. ${ }^{1}$ This case study depicts the events occurring over the years in the life of this young girl whose symptoms started at a very young age with disastrous sequalae leading to her early demise. Autopsy was not done, however, the fungal markers, including galactomannan and alpha-D-glucan, were strongly positive suggesting an aspergillus infection. Primarily, aspergillus species cause invasive disease among immunocompromised patients and most commonly aspergillus fumigatus is isolated. Aspergillus is angio-invasive and it is most commonly disseminated from the lung. Initially, it causes infectious vasculopathy resulting in acute infarction or hemorrhage and later on it spreads into surrounding areas to cause cerebritis and rarely evolves into an abscess..$^{15} \mathrm{~A}$ retrospective study done to find cause of death in patients with HIES reported two patients having disseminated as- pergillus infection involving central nervous system. All the study subjects had cystic lung disease at the time of death as reported in this case. Five out of six patients were infected with aspergillus fumigatus at some point in their life which led to their deaths. ${ }^{14}$ The diagnostic test for neuro-aspergillosis includes PCR assay, tissue histopathology, tissue culture, CSF galactomannan, beta D-glucan levels, and neuroimaging. CSF examination is usually not useful in diagnosis of aspergillosis involving the central nervous system; although, it can be used to rule out other opportunistic infections. CSF galactomannan level can be a useful tool to detect neuro-aspergillosis. In a case study, the positive predictive value of CSF galactomannan in patients with cerebral aspergillosis is approximately $94 \%$. Additionally, raised CSF galactomannan is associated with poor prognosis in patients with severe disease at the time of admission. Moreover, the species aspergillus fumigatus is associated with higher mortality. The most common neuroimaging finding was brain abscess which could be single or multiple ring-enhancing lesions and were observed in around $70 \%$ of the patients as reported in this case. The average duration of treating aspergillosis with voriconazole is six months in patients who survived. In this case study, the patient received voriconazole twice for six months and showed significant improvement each time, thus demonstrating the efficacy of this drug in treating fungal infections especially aspergillosis in HIES patients. ${ }^{16}$ Despite the interventions, the patient could not survive due to extensive disseminated fungal infection superimposed on her primary immunodeficiency.

\section{CONCLUSION}

This case of Hyper IGE syndrome is unusual for two reasons. Firstly, it is a rare immunodeficiency syndrome and about 200 of such cases have been reported in medical literature so far. Secondly, no such case of brain abscess due to fungal etiology is reported in literature up till now. This case study entails the prompt use of neuro-imagining and CSF galactomannan levels for timely diagnosis and management. Yet, a lot has to be discovered about this primary immunodeficiency. However, this case will add to literature the fungal sequalae superimposed in patients with this syndrome and will help to devise protocols in the management of such mycotic infections.

\section{REFERENCES}

1. Freeman AF, Holland SM. The Hyper-IgE Syndromes. Immunol Allergy Clin North Am 2008 May;28(2):277-91. https://doi.org/10.1016/j. iac.2008.01.005

2. Autosomal dominant hyper IgE syndrome-NORD (National Organization for Rare Disorders). Available from: https://rarediseases.org/rare-diseases/ autosomal-dominant-hyper-ige-syndrome/ 
3. Jiao H, Toth B, Erdos M, Fransson I, Rakoczi E, Balogh I, et al. Novel and recurrent STAT3 mutations in hyper-lgE syndrome patients from different ethnic groups. Mol Immunol 2008 Nov;46(1):202-6. https://doi.org/10.1016/j.molimm.2008.07.001

4. Hill HR, Quie PG, Pabst HF, Ochs HD, Clark RA, Klebanoff SJ, et al. Defect in neutrophil granulocyte chemotaxis in job's syndrome of recurrent "cold" staphylococcal abscesses. Lancet 1974 Sep;304(7881):617-9. https://doi.org/10.1016/ S0140-6736(74)91942-4

5. Mogensen TH. STAT3 and the Hyper-lgE syndrome. JAK-STAT 2013 Apr;2(2):e23435. https:// doi.org/10.4161/jkst.23435

6. Ochs HD, Oukka M, Torgerson TR. TH17 cells and regulatory $T$ cells in primary immunodeficiency diseases. J Allergy Clin Immunol 2009 May;123(5):977. https://doi.org/10.1016/j. jaci.2009.03.030

7. Grimbacher B, Holland SM, Puck JM. Hyper-lgE syndromes. Immunol Rev 2005 Feb;203:244-50. https://doi.org/10.1111/j.0105-2896.2005.00228.x

8. Chandesris MO, Melki I, Natividad A, Puel A, Fieschi C, Yun L, et al. Autosomal dominant STAT3 deficiency and hyper-IgE syndrome: Molecular, cellular, and clinical features from a french national survey. Med (United States) $2012 \mathrm{Jul}$;91 (4). https://doi.org/10.1097/MD.0b013e31825f95b9

9. Freeman AF, Collura-Burke CJ, Patronas NJ, llcus LS, Darnell D, Davis J, et al. Brain abnormalities in patients with hyperimmunoglobulin E Syndrome. Pediatrics 2007 May;119(5):e1121-5. https://doi. org/10.1542/peds.2006-2649
10. Ananth Ramakrishnan K, Levin M, Faust SN. Bacterial meningitis and brain abscess. Medicine (Baltimore) 2009 Nov;37(11):567-73. https://doi. org/10.1016/j.mpmed.2009.08.010

11. Alyasin S, Amin R, Teymoori A, Houshmand H, Houshmand G, Bahadoram M. Brain abscess and keratoacanthoma suggestive of hyper $\operatorname{lgE}$ syndrome. Case Reports Immunol 2015;2015:1-5. https://doi.org/10.1155/2015/341898

12. Gatz $S A$, Benninghoff $U$, Schütz $C$, Schulz $A$, Hönig $M$, Pannicke $U$, et al. Curative treatment of autosomal-recessive hyper-IgE syndrome by hematopoietic cell transplantation. Bone Marrow Transplant 2011 Apr;46(4):552-6. https://doi. org/10.1038/bmt.2010.169

13. Metin $A$, Uysal $G$, Güven $A$, Unlu A, Öztürk $M H$. Tuberculous brain abscess in a patient with hyper IgE syndrome. Pediatr Int 2004 Feb;46(1):97-100. https://doi.org/10.1111/j.1328-0867.2004.01845.x

14. Freeman AF, Kleiner DE, Nadiminti H, Davis J, Quezado M, Anderson V, et al. Causes of death in hyper-lgE syndrome. J Allergy Clin Immunol 2007;119(5):1234-40. https://doi.org/10.1016/j. jaci.2006.12.666

15. Lucantoni D, Galzio R, Zenobii M, Magliani V, Sciarra G, D'Arrigo C. Right occipital crebral abscess caused by aspergillus fumigatus. J Neurosurg Sci 1987 Jan-Mar;31(1):29-31.

16. Meena DS, Kumar D, Bohra GK, Kumar G. Clinical manifestations, diagnosis, and treatment outcome of CNS aspergillosis: A systematic review of 235 cases. Infect Dis Now 2021; (xxxx). https:// doi.org/10.1016/j.idnow.2021.04.002

\section{CONFLICT OF INTEREST \\ Authors declare no conflict of interest. GRANT SUPPORT AND FINANCIAL DISCLOSURE None declared.}

\begin{tabular}{|c|c|}
\hline \multicolumn{2}{|c|}{ AUTHORS' CONTRIBUTION } \\
\hline The following authors have made subst & lal contributions to the manuscript as under: \\
\hline Conception or Design: & $\mathrm{NI}, \mathrm{HH}$ \\
\hline Acquisition, Analysis or Interpretation of Data: & NI, HH, IUR, SDK, OSK \\
\hline Manuscript Writing \& Approval: & $\mathrm{NI}, \mathrm{HH}, \mathrm{IUR}, \mathrm{SDK}, \mathrm{OSK}$ \\
\hline
\end{tabular}

